\title{
The Phenomenon of Albumin-Mediated Hepatic Uptake of Organic Anion Transport Polypeptide Substrates: Prediction of the In Vivo Uptake Clearance from the In Vitro Uptake by Isolated Hepatocytes Using a Facilitated-Dissociation Model $^{\text {[ }}$
}

\author{
Seiji Miyauchi, Masayuki Masuda, Soo-Jin Kim, ${ }^{1}$ Yuudai Tanaka, Kyeong-Ryoon Lee, ${ }^{2}$ \\ Shouko Iwakado, Momoko Nemoto, Shotaro Sasaki, Kazumi Shimono, Yoshio Tanaka, \\ and Yuichi Sugiyama
}

Faculty of Pharmaceutical Sciences, Toho University, 2-2-1 Miyama, Funabashi, Chiba, Japan (S.M., M.M., Y.T., S.I., M.N., S.S., K.S., Y.T.) and Sugiyama Laboratory, RIKEN Innovation Center, RIKEN Cluster for Industry Partnerships, RIKEN, 1-7-22

Suehiro-cho, Tsurumi-ku, Yokohama, Japan (S.-J.K., K.-R.L., Y.S.)

Received June 21, 2017; accepted December 11, 2017

\section{ABSTRACT}

The effects of bovine serum albumin and human serum albumin on the unbound hepatic uptake clearance $\left(P S_{u, i n f}\right)$ of the organic aniontransporting polypeptide substrates 1-anilino-8-naphthalene sulfonate (ANS) and pitavastatin (PTV) were determined using primary cultured rat hepatocytes and isolated human hepatocytes, respectively. The $P S_{\mathrm{u} \text {,inf }}$ value of hepatocytes was estimated by dividing the initial uptake rate of these anions by their unbound concentrations. The $P S_{u, i n f}$ values for ANS and PTV were enhanced in the presence of albumin, thereby demonstrating the phenomenon of "albuminmediated" hepatic uptake. We previously constructed a "facilitated-dissociation" model, in which the interaction of the ligand-albumin complex with the cell surface enhanced the dissociation of that complex to provide unbound ligand for uptake to the hepatocytes [J Pharmacokinet Biopharm 16:165-181 (1988)]. That model was able to describe accurately the relationship between the enhancement of the $P S_{u, i n f}$ values and the albumin concentration. By considering the enhancement of hepatic uptake clearance by albumin using this facilitateddissociation model, we could predict accurately the $P S_{u, i n f}$ in vivo from that obtained in isolated hepatocytes. In the light of these findings, we suggest that the facilitated-dissociation model is applicable to describing the phenomenon of albumin-mediated hepatic uptake via organic anion transporters and to evaluating hepatic uptake clearance in vivo.

\section{Introduction}

The liver efficiently eliminates a variety of endogenous and exogenous organic anions from the blood circulation, which then undergo metabolism and/or biliary excretion in the liver. The hepatic uptake process is the most important step in their overall disposition by the liver. According to the conventional "free-drug" hypothesis, it has been assumed that the level of unbound ligand governs the hepatic uptake rate (Wilkinson and Shand, 1975; Shand et al., 1976). However,

This work was supported by Grant-in-Aid for Scientific Research (C) and (S) from the Japanese Ministry of Education, Culture, Sports, Science, and Technology [26460044] and [24229002], respectively.

${ }^{1}$ Current affiliation: Drug Evaluation Group, R\&D Center, CJ HealthCare, Icheon, Korea.

${ }^{2}$ Current affiliation: Life Science Institute, Daewoong Pharmaceutical, Yongin, Korea.

https://doi.org/10.1124/dmd.117.077115.

S This article has supplemental material available at dmd.aspetjournals.org. it is a distinguishing feature of the liver that the hepatic uptake of organic anions is very efficient despite these organic anions being avidly bound to serum albumin, a phenomenon referred to as "albumin-mediated" hepatic uptake (Weisiger et al., 1981; Forker and Luxon, 1983; Bass and Pond, 1988; Tsao et al., 1988b; Meijer and van der Sluijs, 1989; Ichikawa et al., 1992). Consequently, it has been argued that the hepatic uptake of organic anions is more efficient than can be accounted for by their unbound concentration and the free-drug hypothesis.

Ample evidenc $3 \mathrm{e}$ of the efficient elimination of organic anions has led to the proposal of several models to explain the efficient hepatic uptake. These include: 1) the dissociation of ligand-albumin complexes catalyzed by their interaction with the cell surface (Forker and Luxon, 1981; Weisiger et al., 1981; Forker et al., 1982; Forker and Luxon, 1983; Tsao et al., 1988a), 2) the rate-limiting dissociation of ligand-albumin complexes in the extracellular fluid (Weisiger et al., 1984; Weisiger, 1985; van der Sluijs et al., 1987), and 3) the rate-limiting diffusion of ligand through the unstirred water layer adjacent to the cell surface (Bass and Pond, 1988; Weisiger et al., 1991; Ichikawa et al., 1992; Pond et al.,

ABBREVIATIONS: ANS, 1-anilino 8-naphthalene sulfonate; BSA, bovine serum albumin; HSA, human serum albumin; IVIVE, in vitro-to-in vivo extrapolation; $P S_{\text {inf }}$, uptake clearances for total ligand concentration; $P S_{u, i n f}$, uptake clearances for unbound ligand concentration; PTV, pitavastatin. 
1992). The albumin-mediated hepatic uptake effect has been demonstrated previously using various experimental systems, including isolated hepatocytes (Weisiger et al., 1981; Tsao et al., 1988b; Burczynski et al., 2001), primary cultured hepatocytes (Burczynski et al., 1989), isolated perfused liver (Forker and Luxon, 1981, 1983; Tsao et al., 1988a), and isolated basolateral membrane vesicles (Blitzer and Lyons, 1985), also suggesting that the albumin-mediated effect might be driven by a general mechanism, irrespective of species and experimental systems.

Recently, the development of the quantitative in vitro-to-in vivo extrapolation (IVIVE) method has allowed prediction of the uptake clearance in vivo from the uptake rate obtained in human cryopreserved hepatocytes, and the parameters estimated using this technique have been extensively applied to the prediction of human pharmacokinetics. IVIVE of the hepatic uptake clearance with cryopreserved human hepatocytes provided good predictions of the uptake of some organic anions (Watanabe et al., 2010, 2011; Kimoto et al., 2012). However, for other organic anions that tend to bind avidly to serum albumin, the accuracy of the predictions was poor (Watanabe et al., 2010, 2011). It is possible that the albumin-mediated hepatic uptake mechanism is responsible for these poor predictions by IVIVE, because the uptake by human hepatocytes has usually been determined in the absence of serum albumin. Recently, Poulin et al. developed an adjustment method for estimating quantitatively the unbound fraction on the surface of hepatocytes by using the plasma-to-whole liver concentration ratio of extracellular serum albumin, which improved the predictive accuracy of IVIVE for a variety of drugs (Poulin and Haddad, 2015; Poulin et al., 2016). Therefore, adequate prediction of hepatic uptake clearance by IVIVE requires adjustment for the albumin-mediated uptake factor, the enhancement of hepatic uptake seen in physiologic albumin concentrations.

In the present study, we investigated whether bovine serum albumin (BSA) and human serum albumin (HSA), respectively, enhanced the unbound uptake clearance of 1-anilino-8-naphthalene sulfonate (ANS) by primary cultured rat hepatocytes and of pitavastatin (PTV) by human isolated hepatocytes. Both organic anions have been demonstrated to be taken up efficiently by the liver via organic anion-transporting polypeptides (Oatp/OATP), despite their avid binding to serum albumin in the blood circulation (Sugiyama et al., 1983; Chung et al., 1990a,b; Watanabe et al., 2010, 2011; Kimoto et al., 2012). We demonstrated the role of the albumin-mediated hepatic uptake phenomenon in uptake of these anions by rat and human hepatocytes. Furthermore, we determined whether the relationship between the enhancement of the unbound hepatic clearance and the albumin concentration could be predicted by a "facilitated-dissociation" model constructed previously (Tsao et al., 1988a), in which the interaction of the ligand-albumin complex with the cell surface enhances the dissociation of the complex, resulting in more unbound ligand being available for uptake by hepatocytes.

\section{Materials and Methods}

Materials. Chemicals including ANS, BSA (fraction V), HSA, KrebsHenseleit buffer, and PTV were purchased from Sigma-Aldrich (St. Louis, MO). Cryopreserved human hepatocytes (lot 109) were purchased from BD Biosciences (Woburn, MA). Six-week-old Sprague Dawley (SD) rats were purchased from Sankyo Laboratory Corp. Inc. (Tokyo, Japan). All other chemicals used were of the highest purity available from Wako Chemical Inc. (Tokyo, Japan).

Measurement of ANS Uptake by Primary Cultured Rat Hepatocytes. Hepatocytes were isolated from the livers of 6-week-old male SD rats that were given free access to food and water. The isolation procedure was basically the same as previously reported (Miyauchi et al., 1993; Masuda et al., 2014). Cell viability, determined by Trypan blue exclusion, ranged from 95 to $98 \%$. Cells

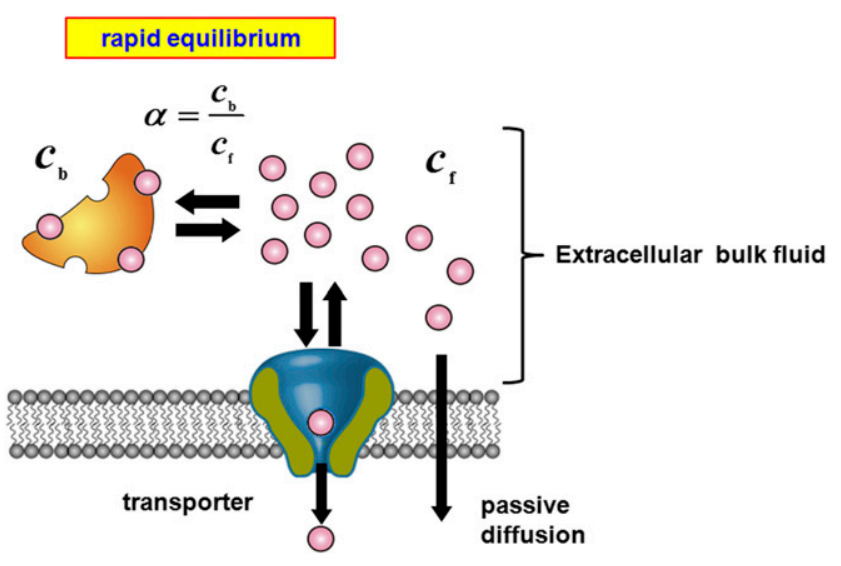

Fig. 1. A conventional model of hepatic uptake via a transporter. In the conventional model, it is assumed that rapid equilibrium in the binding of a ligand to albumin is maintained in the extracellular bulk fluid. The unbound and bound ligands are designated as $c_{\mathrm{b}}$ and $c_{\mathrm{f}}$, respectively. The ratio of $c_{\mathrm{b}}$ to $c_{\mathrm{f}}$ is designated as $\alpha$. The uptake rate of a ligand is presumed to be governed not only by transportmediated uptake but also by passive diffusion uptake (eq. 1).

were suspended at a density of $3.3 \times 10^{5}$ cells $/ \mathrm{ml}$ in William's E medium supplemented with dexamethasone $(5 \mathrm{nM})$, insulin $(10 \mathrm{nM}), 10 \%$ fetal calf serum, $100 \mathrm{IU} / \mathrm{ml}$ penicillin, and $100 \mu \mathrm{g} / \mathrm{ml}$ streptomycin. A 3-ml aliquot of cell suspension was added to each well of a six-well plate. The culture medium was exchanged with fresh medium 4 hours after the initial seeding. Cells were cultured for an additional 16 hours at $37^{\circ} \mathrm{C}$ in $5 \% \mathrm{CO}_{2} / 95 \%$ air and then used for ANS uptake experiments. The primary cultured hepatocytes were used for the uptake experiments only after being cultured for less than 22 hours. The experimental protocol for the use of the animals was approved by Toho University Animal Care and Use Committee (approval number: 16-53-258).

Uptake of ANS in primary cultured hepatocytes was measured at $37^{\circ} \mathrm{C}$ in uptake buffer ( $\mathrm{pH}$ 7.5) as described previously (Masuda et al., 2014). The composition of the uptake buffer was $140 \mathrm{mM} \mathrm{NaCl}, 3 \mathrm{mM} \mathrm{KCl}, 1 \mathrm{mM} \mathrm{CaCl}{ }_{2}$, $1 \mathrm{mM} \mathrm{MgCl}_{2}, 5 \mathrm{mM}$ glucose, and $10 \mathrm{mM}$ Hepes/Tris adjusted to $\mathrm{pH}$ 7.5. Cells in each well were washed with the prewarmed uptake buffer and were preincubated for 5 minutes at $37^{\circ} \mathrm{C}$. After aspiration of the uptake buffer, uptake was initiated by the addition of a 1-ml aliquot of uptake buffer containing ANS and BSA at various concentrations as indicated in the figure legends. At the designated time, the buffer was aspirated, and cells were immediately washed twice with ice-cold uptake buffer. The cells were then dissolved in a 1-ml aliquot of $1 \%$ (w/v) Triton$\mathrm{X}$. The dissolved cells were diluted 10-fold with ethanol, and the samples were centrifuged at $18,000 \mathrm{~g}$ for 10 minutes by a tabletop centrifuge. This aliquot of the supernatant was used to measure ANS concentration. The fluorescence intensity resulting from ANS uptake to the cells was measured at $470 \mathrm{~nm}$ (excitation $370 \mathrm{~nm}$ ) using an Enspire multiplate fluorescence reader (PerkinElmer Inc., Waltham, MA) (Sugiyama et al., 1983; Chung et al., 1990a).

Measurement of PTV Uptake by Suspended Human Hepatocytes. The rate of uptake of PTV by suspended human hepatocytes was determined using cryopreserved human hepatocytes and a centrifugal filtration technique, as described previously (Watanabe et al., 2010, 2011). For the uptake experiments, cryopreserved hepatocytes were thawed in a water bath at $37^{\circ} \mathrm{C}$ then placed on ice, after which they were resuspended in Krebs-Henseleit buffer. Cell viability was determined by Trypan blue exclusion, and the suspension was diluted to $2 \times 10^{6}$ cells $/ \mathrm{ml}$. Then, $150-\mu \mathrm{l}$ aliquots of cells were placed in test plates and prewarmed in a $37^{\circ} \mathrm{C}$ incubator for 3 minutes. Uptake incubations were initiated by the addition of $150 \mu \mathrm{l}$ of prewarmed $10 \mu \mathrm{M}$ PTV, with and without HSA, which resulted in a final substrate concentration of $5 \mu \mathrm{M}$ and a cell density of $1 \times 10^{6}$ cells $/ \mathrm{ml}$ in a $300-\mu 1$ incubation volume. After a designated time $(0.25$ and 1.25 minutes), the reactions were terminated by separating the cells from the medium using a centrifugal filtration technique. For this purpose, the $80-\mu 1$ aliquot of incubation mixture was placed in a $0.4-\mathrm{ml}$ centrifuge tube (Sarstedt, Numbrecht, Germany) containing 50- $\mu \mathrm{l}$ of $5 \mathrm{M}$ ammonium acetate under a 100$\mu l$ layer of an oil mixture (density 1.05, mixture of silicone oil and mineral oil; Sigma-Aldrich). Samples were then centrifuged for 10 seconds in a microfuge 


\section{Bound ligand}

Unbound ligand

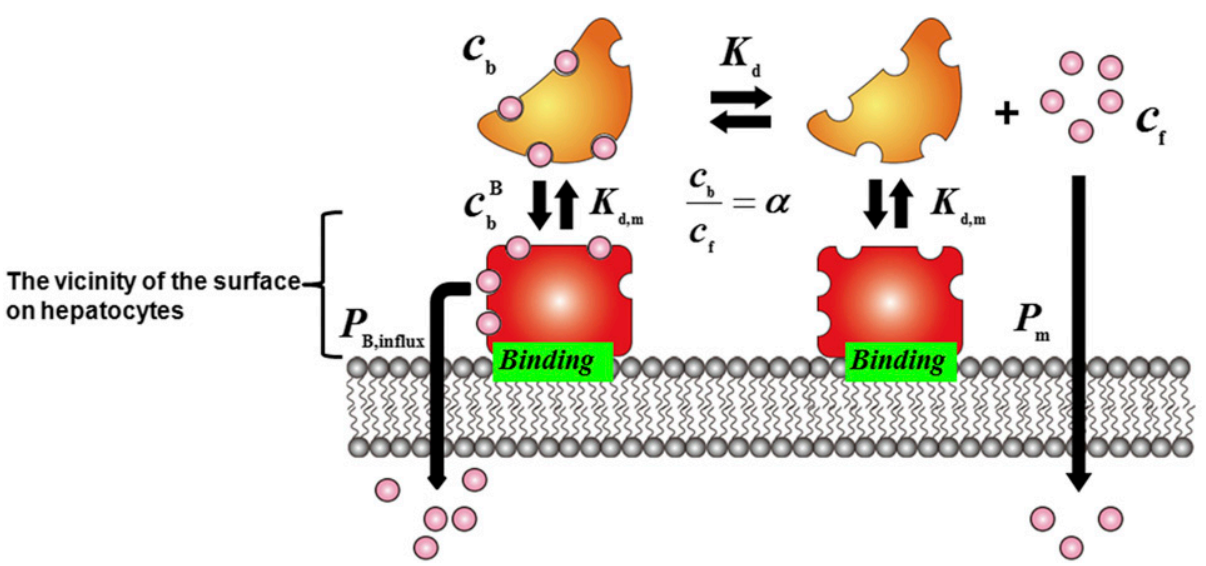

Fig. 2. The facilitated-dissociation model. The uptake of highly albumin-bound ligands to hepatocytes comprises a pathway for the unbound ligands and an additional pathway for the albumin-bound ligands. The interaction of the ligand-albumin complex with the surface of hepatocytes makes more unbound ligand available for uptake than would be expected according to the conventional model. It was assumed that the unbound and ligandbound albumin compete for the same binding site(s) on the surface of hepatocytes with the same affinity (dissociation constant, $K_{\mathrm{d}, \mathrm{m}}$ ).

(Beckman Coulter, Brea, CA). During centrifugation, the hepatocytes rapidly passed through the oil layer into the aqueous solution (5 M ammonium acetate). The tubes were frozen in liquid nitrogen immediately after centrifugation and stored at $-20^{\circ} \mathrm{C}$ until analysis. The bottom of the tube was sliced off, and the contents were transferred to a new tube. Protein in the aqueous solution was precipitated with three volumes of acetonitrile/methanol $(50 / 50, \mathrm{v} / \mathrm{v})$ and removed by centrifugation at $15,000 \mathrm{~g}$ at $4^{\circ} \mathrm{C}$ for 10 minutes. The supernatants from the top (medium) and the bottom (cells) of the tube were analyzed by liquid chromatography coupled with tandem mass spectrometry.

PTV was analyzed on a Shimadzu LCMS-8050 triple quadrupole mass spectrometer (Shimadzu, Kyoto, Japan). Samples (5 $\mu$ l) were injected onto a Kinetex C18 column $(2.1 \times 100 \mathrm{~mm}, 2.6 \mu \mathrm{m}$; Phenomenex, Torrance, CA $)$ and eluted by a mobile phase with initial conditions of $5 \%$ solvent $\mathrm{B}$, followed by a linear gradient of $5 \%$ solvent B to $80 \%$ solvent B over 3.0 minutes (solvent A, $0.1 \%$ formic acid in water; solvent $\mathrm{B}, 100 \%$ acetonitrile) at a flow rate of $0.3 \mathrm{ml} / \mathrm{min}$. The column and autosampler tray temperature were maintained at 40 and $4^{\circ} \mathrm{C}$, respectively. For PTV, the mass spectrometer was operated in positive turbo-ion-spray mode at the transitions $(\mathrm{m} / \mathrm{z})$ 422-290.

Measurement of ANS Binding to BSA by the Equilibrium Dialysis Method. A dialysis cell comprising two chambers was divided by a $15-\mathrm{kDa}$ cutoff dialysis membrane (Spectrum Medical Industries. Inc., Los Angeles, CA). Five milliliters of uptake buffer with or without added BSA was put into each chamber. ANS was added to the side containing BSA. After equilibrium was achieved at $37^{\circ} \mathrm{C}$ (more than 22 hours), the aliquot of the sample in each cell was diluted more than 10-fold with EtOH, and ANS concentrations were measured as described above. The recovery of ANS was greater than $98 \%$.

Measurement of PTV Binding to HSA by the Equilibrium Dialysis Method. The binding of PTV to HSA was determined using a single-use RED (rapid equilibrium dialysis) device (Thermo Scientific, Rockford, IL). In this study, PTV was added at a final concentration of $5 \mu \mathrm{M}$ to phosphate-buffered saline (PBS) buffer, $\mathrm{pH} 7.4$, containing HSA at $0,0.125,0.25,0.5$, and $1 \%$, and then incubated at $37^{\circ} \mathrm{C}$ for 24 hours on an orbital shaker. According to the manufacturer's instructions, $300 \mu \mathrm{l}$ of the mixture was placed into the sample chamber, and the dialysis buffer (i.e., PBS, pH 7.4) was placed into the buffer chamber. After equilibrium was achieved (i.e., after 20 hours), the concentrations of PTV in both chambers were measured using liquid chromatography coupled with tandem mass spectrometry analysis, as described above. The recovery of PTV was approximately 92-98\%.

Kinetic Analyses. The initial uptake rates were calculated from the slopes of the time courses from 1 to 3 minutes for ANS and from 0.25 to 1.25 minutes for PTV, using linear regression analysis. The uptake clearances $\left(P S_{\mathrm{u}, \text { inf }}\right.$ and $\left.P S_{\mathrm{inf}}\right)$ for unbound and total ligand concentrations were estimated by dividing the initial uptake rate by the unbound and total ligand concentrations, respectively. Saturation kinetics of the initial uptake rates were analyzed by fitting the concentration dependency of the initial uptake rate of ANS to a MichaelisMenten-type equation with a saturable component. The kinetic parameters $\left(V_{\max }\right.$, the maximum uptake rate; $K_{\mathrm{m}}$, Michaelis constant) were determined by an iterative nonlinear least-squares method using Sigmaplot (v.13; Systat Software Inc., San Jose, CA).

Kinetic Modeling. We used two models in this study, a conventional freedrug model and the facilitated-dissociation model (Tsao et al., 1988a). The models used here assume that the association and dissociation of ligand and albumin were rapid enough to achieve equilibrium. The two models are summarized below.

A Conventional Model of Hepatic Uptake Involving a Transporter. In the conventional free-drug model (Fig. 1), it is assumed that rapid equilibrium of the binding of a ligand to albumin can be achieved in the extracellular bulk fluid. The hepatic uptake of the ligand is assumed to comprise transporter-mediated uptake and passive diffusion across the membrane, which correspond to the saturable and nonsaturable components, respectively, in the Michaelis-Menten equation. The uptake rate is described by the following equation:

$$
v=\frac{V_{\max } \cdot c_{\mathrm{f}}}{K_{\mathrm{m}}+c_{\mathrm{f}}}+P_{\mathrm{d}} \cdot c_{\mathrm{f}}
$$

A

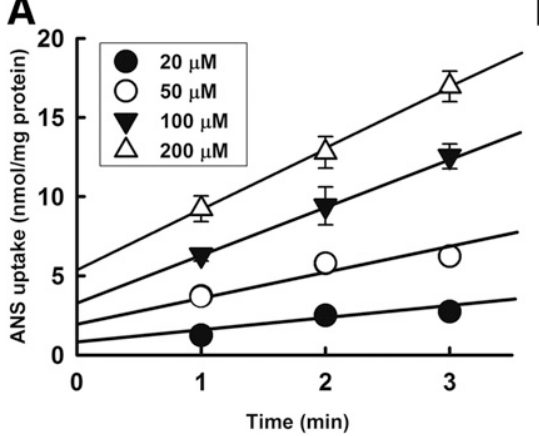

B

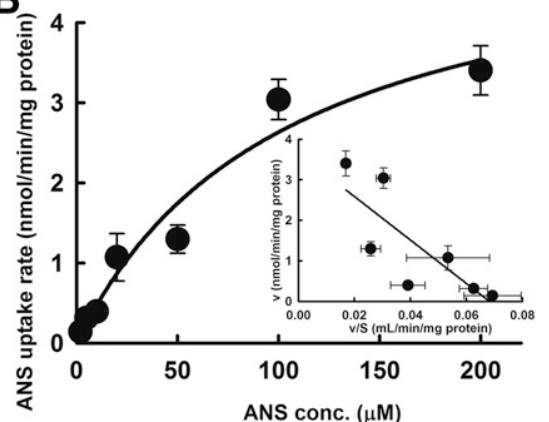

Fig. 3. Concentration-dependency of ANS uptake by primary cultured rat hepatocytes. (A) Time course of ANS uptake by rat hepatocytes. The uptake rate of ANS was measured for 3 minutes at $37^{\circ} \mathrm{C}$ and $\mathrm{pH} 7.5$. Each point represents the mean \pm S.E. $(n=$ 4). (B) Concentration-dependency of the rate of uptake of ANS. The rate of uptake of ANS was measured for 3 minutes at $37^{\circ} \mathrm{C}$ and $\mathrm{pH} 7.5$ in the presence of increasing ANS concentrations (2$200 \mu \mathrm{M})$. The inset figure shows the Eadie-Hofstee plot of ANS uptake. Lines fitted to the nonlinear or linear forms of the Michaelis-Menten equation are shown. $K_{\mathrm{m}}$ (Michaelis constant) and $V_{\max }$ (maximal uptake rate) were estimated to be $53.7 \pm$ $18.2 \mu \mathrm{M}$ and $3.7 \pm 0.8 \mathrm{nmol} / \mathrm{min}$ per milligram protein, respectively ( \pm calculated S.D.). 


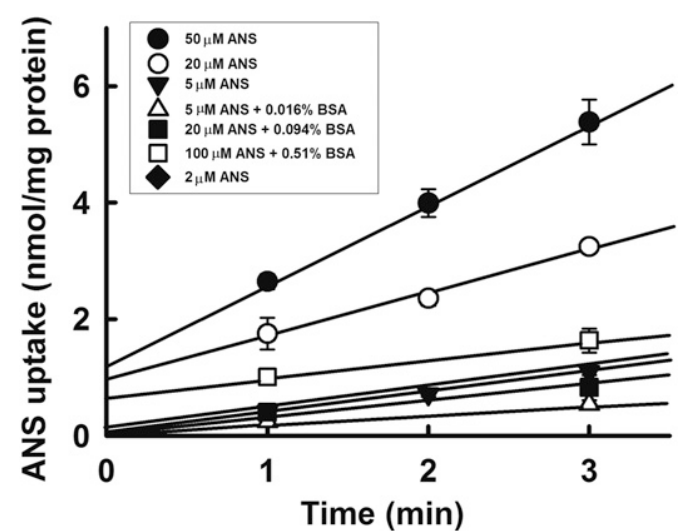

Fig. 4. Effect of BSA on ANS uptake by primary cultured rat hepatocytes. The uptake of ANS was measured for 3 minutes at $37^{\circ} \mathrm{C}$ and $\mathrm{pH}$ 7.5. Each point represents the mean \pm S.E. $(n=4)$.

where $c_{\mathrm{f}}$ is the unbound ligand in the extracellular fluid; $V_{\max }$ and $K_{\mathrm{m}}$ represent the maximum uptake rate and Michaelis constant, respectively; and $P_{\mathrm{d}}$ is the passive diffusion coefficient. Under linear conditions of transporter kinetics, eq. 1 can be reduced to

$$
v=\left(\frac{V_{\max }}{K_{\mathrm{m}}}+P_{\mathrm{d}}\right) \cdot c_{\mathrm{f}}
$$

In the initial uptake by hepatocytes, the following mass-balance equation holds:

$$
c_{0} V_{\mathrm{ext}}=\left(c_{\mathrm{b}}+c_{\mathrm{f}}\right) V_{\mathrm{ext}}=(\alpha+1) c_{\mathrm{f}} V_{\mathrm{ext}}
$$

where $c_{0}$ and $c_{\mathrm{b}}$ represent the concentrations for the total ligand and the ligand bound to albumin, respectively; the ratio of $c_{\mathrm{b}}$ to $c_{\mathrm{f}}$ is designated as $\alpha$, and $V_{\mathrm{ext}}$ is the extracellular fluid volume. Substituting eq. 3 into eq. 2 gives:

$$
\mathrm{v}=\left(\frac{V_{\max }}{K_{\mathrm{m}}}+P_{\mathrm{d}}\right) \cdot \frac{1}{1+\alpha} \cdot c_{0}
$$

The uptake clearances for the total ligand concentration $\left(P S_{\text {inf }}\right)$ and the unbound ligand concentration $\left(P S_{\mathrm{u}, \mathrm{inf}}\right)$ can be described as follows.

$$
\begin{gathered}
P S_{\text {inf }}=\left(\frac{V_{\max }}{K_{\mathrm{m}}}+P_{\mathrm{d}}\right) \cdot \frac{1}{1+\alpha} \\
P S_{\mathrm{u}, \text { inf }}=\left(\frac{V_{\max }}{K_{\mathrm{m}}}+P_{\mathrm{d}}\right)
\end{gathered}
$$

The ratio $(R)$ of $P S_{\mathrm{u}, \text { inf }}$ in the presence of albumin $\left(P S_{\mathrm{u}, \text { inf }}(+)\right)$ to that in the absence of albumin $\left(P S_{\mathrm{u}, \text { inf }}(-)\right)$ can be derived from eq. 6 :

$$
R=\frac{P S_{\mathrm{u}, \text { inf }}(+)}{P S_{\mathrm{u}, \mathrm{inf}}(-)}=1
$$

where the $R$ value in the conventional model usually represents 1 , irrespective of the presence of albumin.

A Facilitated-Dissociation Model of the Dissociation of Unbound Ligand from the Ligand-Albumin Complex. In our previous study, the albuminmediated hepatic transport phenomenon was characterized using isolated hepatocytes and isolated perfused liver and was analyzed using a facilitated-dissociation model in which the interaction of the ligand-albumin complex with the surface of hepatocytes enhances dissociation of the ligand from the complex (Tsao et al., 1988a,b). As shown in Fig. 2, the mechanism of this facilitation has been postulated to be an interaction between the ligand-albumin complex and the surface of hepatocytes that induces a conformational change in albumin, which results in enhancement of the dissociation of ligand from the ligand-albumin complex near the cell surface and consequently effective uptake of the ligand (Forker and Luxon, 1981, 1983; Tsao et al., 1988a). If we presume that the unbound albumin and the ligand-bound albumin compete for the same binding sites on the surface of hepatocytes, the fraction of albumin bound to the surface of the hepatocytes $(\lambda)$ is given by the following equation:

$$
\lambda=\frac{B_{\max }}{K_{\mathrm{d}, \mathrm{m}}+[A l b]}
$$

where $B_{\max }$ and $K_{\mathrm{d}, \mathrm{m}}$ represent the capacity of albumin binding sites on the surface of hepatocytes and the dissociation constant of the bound albumin from the surface, respectively, and $[A l b]$ represents the concentration of albumin. The governing equations for the uptake rate of ligand (v) derived by Tsao et al. (1988a) are as follows:

$$
\begin{gathered}
v=P S_{\mathrm{inf}} \cdot c_{0}=P S_{\mathrm{u}, \text { inf }} \cdot f_{\mathrm{u}} \cdot c_{0}+P S_{\mathrm{b}, \text { inf }} \cdot \lambda \cdot\left(1-f_{\mathrm{u}}\right) \cdot c_{0} \\
P S_{\mathrm{u}, \text { inf }}(+)=P S_{\mathrm{u}, \text { inf }}+P S_{\mathrm{b}, \text { inf }} \cdot \lambda \cdot\left(\frac{1}{f_{\mathrm{u}}}-1\right)
\end{gathered}
$$

where $c_{0}$ represents the total concentration of ligand; $P S_{\mathrm{inf}}, P S_{\mathrm{u}, \mathrm{inf}}(+), P S_{\mathrm{u}, \mathrm{inf}}$, and $P S_{\mathrm{b}, \text { inf }}$ represent the uptake clearances for the total and unbound ligands in the presence of albumin, the permeability clearances for the unbound ligand, and the unbound ligand dissociated from the ligand-albumin complex at the surface, respectively; $f_{\mathrm{u}}$ represents the fraction of the unbound ligand in the extracellular fluid. $P S_{\mathrm{u}, \text { inf }}$ and $P S_{\mathrm{b}, \text { inf }}$ values include the transporter-mediated transport and passive diffusion. The $f_{\mathrm{u}}$ value is expressed as the following equation:

$$
f_{\mathrm{u}}=\frac{1}{1+\frac{n[\mathrm{Alb}]}{K_{\mathrm{d}}}}
$$

where $n$ and $K_{\mathrm{d}}$ represent the number of binding sites on the albumin and the dissociation constant of the ligand, respectively.

The ratio $(R)$ of $P S_{\mathrm{u}, \mathrm{inf}}$ in the presence of albumin $\left(P S_{\mathrm{u}, \mathrm{inf}}(+)\right)$ to that in the absence of albumin $\left(P S_{\mathrm{u}, \text { inf }}(-)\right)$ can be derived from eqs. 8,10 , and 11 , as follows:

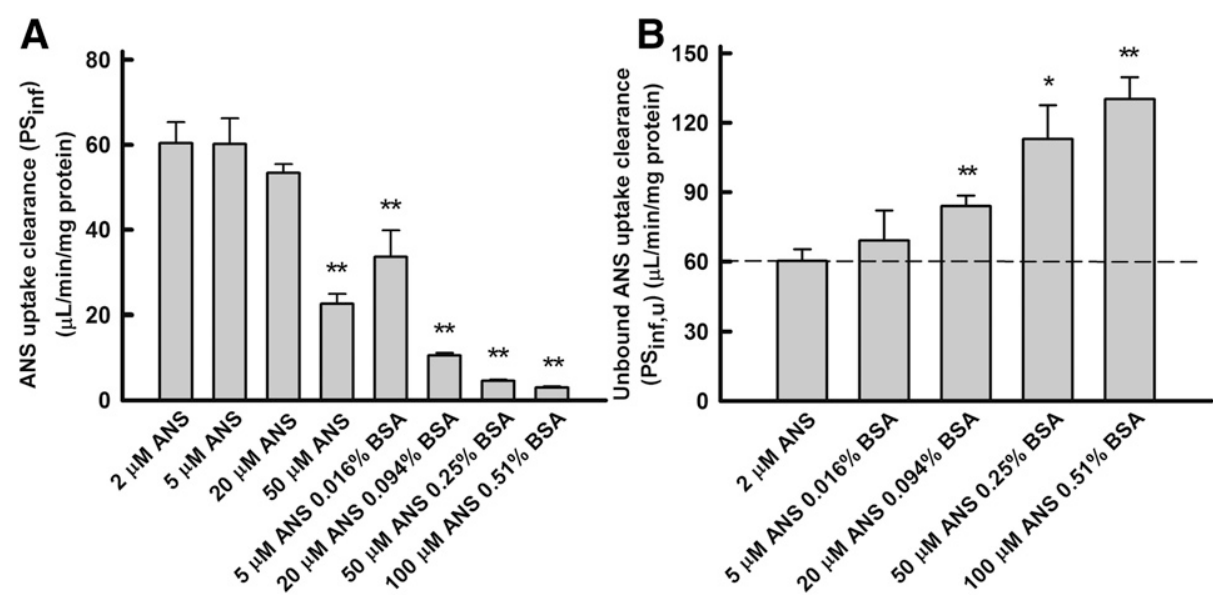

Fig. 5. The effect of BSA on unbound uptake clearance $\left(\mathrm{PS}_{\mathrm{u}, \mathrm{inf}}\right)$ of ANS by rat hepatocytes. (A) Uptake clearance $\left(P S_{\text {inf }}\right)$ of ANS. The uptake clearance was estimated by dividing the uptake rate by the ANS concentration in the medium (Fig. 4). (B) Unbound uptake clearance $\left(P S_{\mathrm{u}, \mathrm{inf}}\right)$ of ANS. The values of $P S_{\mathrm{u}, \mathrm{inf}}$ were estimated by normalizing the value of $P S_{\text {inf }}(\mathrm{A})$ to the unbound fraction of ANS. The unbound concentration of ANS in the presence of BSA was kept at $2.4 \mu \mathrm{M}$. Enhancement of the unbound uptake clearance of ANS was saturated at higher concentrations of BSA. Comparison of the uptake clearances in the presence and absence of albumin were performed with Student's $t$ test. $* P<$ 0.05 (significantly different from control); $* * P<$ 0.01 (significantly different from control). 
TABLE 1

Relationship between BSA concentration (unbound fraction) and $\mathrm{PS}_{\mathrm{u}, \text { inf }}$ of ANS

\begin{tabular}{lccccc}
\hline \multicolumn{1}{c}{ BSA (\%) } & 0 & 0.016 & 0.094 & 0.25 & 0.51 \\
\hline Unbound fraction & 1 & 0.486 & 0.126 & 0.0487 & 0.0231 \\
$P S_{\mathrm{u} \text {,inf }}{ }^{a}(\mu \mathrm{l} / \mathrm{min}$ per milligram protein) & $60.3 \pm 5.0$ & $69.1 \pm 12.9$ & $84.0 \pm 4.6$ & $113 \pm 15$ & $130 \pm 10$ \\
$P S_{\mathrm{u}, \text { inf }}(\mu \mathrm{l} / \mathrm{min}$ per milligram protein $)$ & $62.7 \pm 1.4$ & $83.9 \pm 0.4$ & $120 \pm 14$ & $171 \pm 19$ & $159 \pm 33$ \\
\hline
\end{tabular}

${ }^{a}$ Data shown in Fig. 5. ANS hepatic uptakes were determined as ANS and BSA concentrations were varied, and the unbound concentration of ANS was kept constant at approximately $2.4 \mu \mathrm{M}$.

${ }^{b}$ Data shown in Fig. 6. ANS uptakes were determined with varying BSA concentrations and keeping ANS concentration constant at $20 \mu \mathrm{M}$.

$$
R=\frac{P S_{\mathrm{u}, \text { inf }}(+)}{P S_{\mathrm{u}, \text { inf }}(-)}=1+\frac{P S_{\mathrm{b}, \text { inf }}}{P S_{\mathrm{u}, \text { inf }}} \cdot\left(\frac{1}{f_{\mathrm{u}}}-1\right) \cdot \lambda=1+\frac{\frac{P S_{\mathrm{b}, \mathrm{inf}}}{P S_{\mathrm{u}, \mathrm{inf}}} \cdot B_{\mathrm{max}}[A l b] \cdot \frac{n}{K_{\mathrm{d}}}}{K_{\mathrm{d}, \mathrm{m}}+[A l b]}
$$

where $\frac{P S_{\text {binf }}}{P S_{\text {unf }}} \cdot B_{\text {max }}$ represents the parameters with regard to the relative capacity for the interaction of the albumin with the surface of hepatocytes and is designated as $r \cdot B_{\max }$.

Alternatively, we also analyzed the obtained data using the model derived by Forker et al. (Forker and Luxon, 1983), which was categorized as a facilitateddissociation model but was more similar to a model whose basis was the "albumin receptor" theory. According to this model, the ratio of $\boldsymbol{P S} \boldsymbol{u}_{\mathrm{u}, \mathrm{inf}}(+)$ to $\boldsymbol{P S _ { \mathrm { u } , \mathrm { inf } }}(-)$ can be expressed, as follows:

$$
R=\frac{P S_{\mathrm{u}, \text { inf }}(+)}{P S_{\mathrm{u}, \text { inf }}(-)}=\frac{1+\left(\frac{1}{f_{\mathrm{u}}}-1\right) \cdot \lambda}{1-\left(1-f_{\mathrm{u}}\right) \cdot \lambda}=\frac{1+[A l b] \cdot \frac{n}{K_{\mathrm{d}}} \cdot \frac{B_{\max }}{B_{\max }+K_{\mathrm{d}, \mathrm{m}}+[A l b]}}{1-\frac{n[A l b]}{K_{\mathrm{d}}+n[A l b]} \cdot \frac{B_{\max }}{B_{\max }+K_{\mathrm{d}, \mathrm{m}}+[A l b]}}
$$

Extrapolation of the In Vivo Hepatic Uptake Clearance from the Uptake Clearance Measured Using Isolated Hepatocytes. The unbound hepatic uptake clearances for ANS and PTV in vivo (per body weight) were scaled up from the in vitro unbound hepatic uptake clearance using the following scaling factors: $1.2 \times 10^{8}$ cells $/ \mathrm{g}$ liver, $40.0 \mathrm{~g}$ liver $/ \mathrm{kg}$ body weight for rat, and $25.7 \mathrm{~g}$ liver $/ \mathrm{kg}$ body weight for human (Davies and Morris, 1993; Miyauchi et al., 1993).

In vivo hepatic overall intrinsic clearance, which represents the elimination of unbound ligands $\left(C L_{\mathrm{h}, \mathrm{u}, \mathrm{int} \text {,all }}^{\text {viv }}\right)$ from circulating blood, was also estimated from the in vivo hepatic clearance using a dispersion model as follows:

$$
\begin{gathered}
C L_{\mathrm{h}, \mathrm{B}}=Q_{\mathrm{h}} \times\left(1-F_{\mathrm{h}}\right) \\
F_{\mathrm{h}}=\frac{4 a}{(1+a)^{2} \cdot \exp \left\{\frac{(a-1)}{2 D_{\mathrm{N}}}\right\}-(1-a)^{2} \cdot \exp \left\{-\frac{(a+1)}{2 D_{\mathrm{N}}}\right\}} \\
a=\left(1+4 R_{\mathrm{N}} \cdot D_{\mathrm{N}}\right)^{1 / 2} \\
R_{\mathrm{N}}=f_{\mathrm{B}} \cdot C L_{\mathrm{h}, \mathrm{u}, \text { int,all }}^{\text {vivo }}
\end{gathered}
$$

where $C L_{\mathrm{h}, \mathrm{B}}, Q_{\mathrm{h}}, D_{\mathrm{N}}$, and $f_{\mathrm{B}}$ are the hepatic clearance, hepatic blood flow rate, the dispersion number, and unbound fraction in the blood, respectively. The $Q_{\mathrm{h}}$ values for rat and human are 55.2 and $20.7 \mathrm{ml} / \mathrm{min}$ per kilogram, respectively (Davies and Morris, 1993). The $D_{\mathrm{N}}$ value was set at 0.17 . The hepatic uptake clearance in vivo was estimated on the basis of eqs. 14-17, using the dispersion model described previously (Roberts and Rowland, 1986; Iwatsubo et al., 1996).

\section{Results}

Initial Uptake Rate of ANS by Primary Cultured Rat Hepatocytes in the Absence and Presence of BSA. The uptake rate of ANS by primary cultured rat hepatocytes in the absence of BSA was measured, and the results are summarized in Fig. 3. The ANS uptake increased linearly over 3 minutes, and the initial uptake rate was determined from the uptake over 1-3 minutes. The relationship between the initial uptake rate and concentration of ANS showed saturation, and the data fit the Michaelis-Menten equation describing a single saturable process; this was supported by the linearity of the Eadie-Hofstee plot shown in the inset. The kinetic parameters $K_{\mathrm{m}}$ (Michaelis-Menten constant) and $V_{\max }$ (maximal uptake rate) were $53.7 \pm 18.2 \mu \mathrm{M}$ and 3.7 $\pm 0.8 \mathrm{nmol} / \mathrm{min}$ per milligram protein, respectively ( \pm calculated S.D.). These estimated $K_{\mathrm{m}}$ and $V_{\max }$ values were similar to those reported for isolated rat hepatocytes (Chung et al., 1990a). The ANS uptake by primary cultured hepatocytes determined in the presence of various concentrations of BSA is summarized in Fig. 4. ANS binds avidly to albumin, and the presence of BSA caused a remarkable decrease in its uptake. The hepatic uptake clearance $\left(P S_{\text {inf }}\right)$ and the unbound uptake clearance $\left(P S_{\mathrm{u}, \text { inf }}\right)$ were estimated by dividing the uptake rate by the initial concentration and unbound concentrations, respectively, as depicted in Fig. 5 and summarized in Table 1. First, to confirm whether the ANS uptake by Oatp was linear within the range of $20 \mu \mathrm{M}$, we determined the ANS uptake rates at various concentrations of ANS. As shown in Fig. 4, the hepatic uptake clearances for ANS in the range of 2$20 \mu \mathrm{M}$ remained unchanged (did not differ significantly), being consistent with the estimated $K_{\mathrm{m}}$ value. We then conducted the uptake experiments under conditions of varying ANS and BSA concentrations at the same time maintaining the ratio of ANS to BSA, or keeping the unbound concentration of ANS constant at approximately $2.4 \mu \mathrm{M}$. These experimental conditions followed those developed by

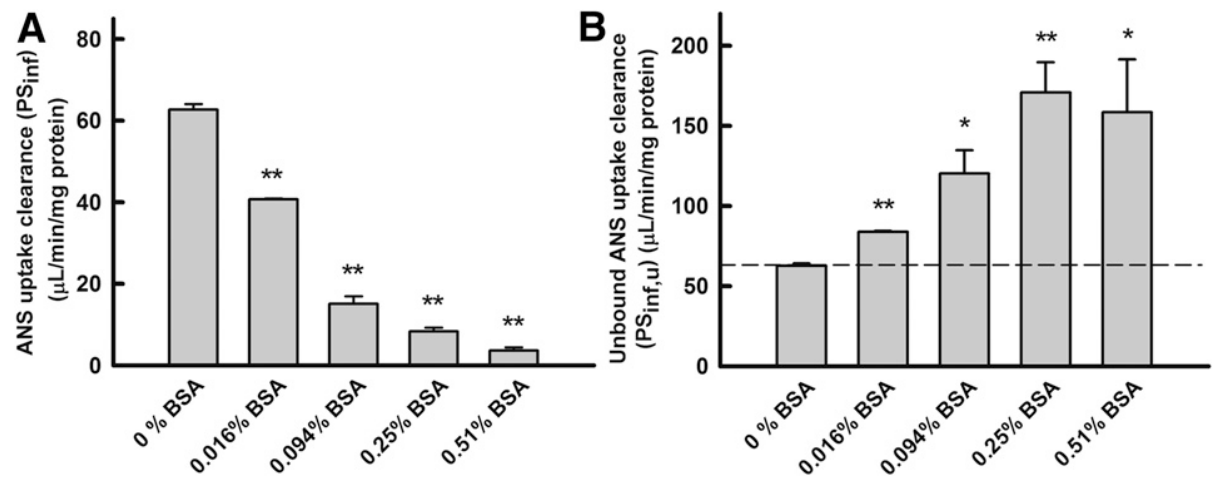

Fig. 6. The effect of BSA on unbound uptake clearance $\left(\mathrm{PS}_{\mathrm{u}, \mathrm{inf}}\right)$ of ANS by rat hepatocytes. (A) Uptake clearance (PS inf $_{\text {) }}$ of ANS. The uptake clearance was estimated by dividing the uptake rate by the $20 \mu \mathrm{M}$ ANS concentration in the medium. (B) Unbound uptake clearance $\left(P S_{\mathrm{u}}\right.$,inf $)$ of ANS. The values of $P S_{\mathrm{u}, \text { inf }}$ were estimated by normalizing the value of $P S_{\text {inf }}$ (A) to the unbound fraction of ANS. Enhancement of the unbound uptake clearance of ANS was saturated at higher concentrations of BSA. Comparison of the uptake clearances in the presence and absence of albumin were performed with Student's $t$ test. $* P<0.05$ (significantly different from control); $* * P<0.01$ (significantly different from control). 

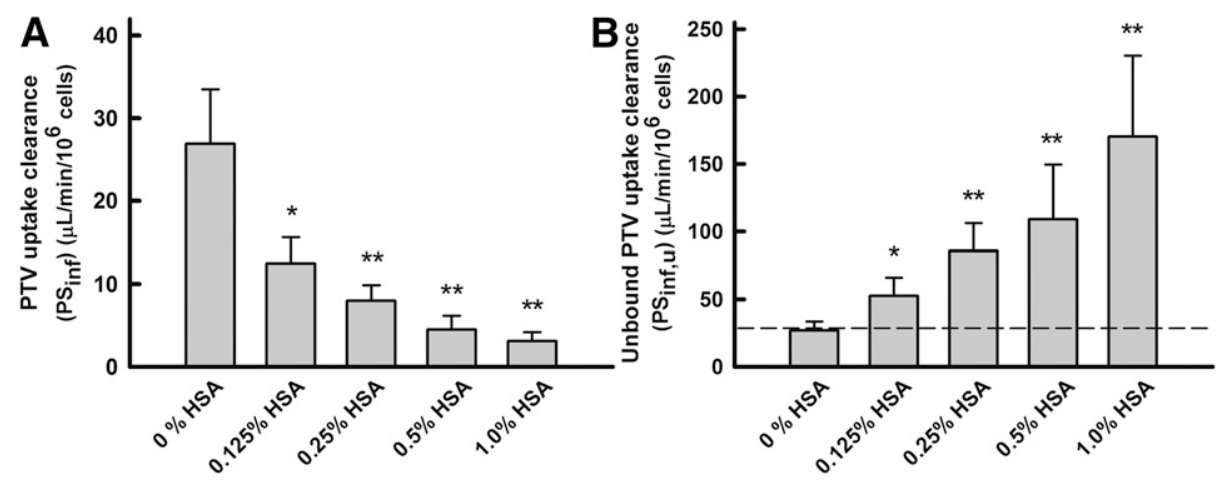

Fig. 7. The effect of HSA on unbound uptake clearance $\left(\mathrm{PS}_{\mathrm{u}, \text { inf }}\right)$ of PTV by human hepatocytes. (A) Uptake clearance $\left(\mathrm{PS}_{\mathrm{inf}}\right)$ of PTV. The uptake rate of $5 \mu \mathrm{M}$ PTV was measured for 75 seconds at $37^{\circ} \mathrm{C}$ and $\mathrm{pH}$ 7.5. The uptake clearance was estimated by dividing the uptake rate by the PTV concentration in the medium. Each bar represents the mean \pm S.E. $(n=4)$. (B) Unbound uptake clearance $\left(P S_{\mathrm{u}, \mathrm{inf}}\right)$ of PTV. The values of $P S_{\mathrm{u}, \mathrm{inf}}$ were estimated by normalizing the value of $P S_{\mathrm{inf}}$ (A) to the unbound fraction of PTV. The enhancement of the unbound uptake clearance of PTV was saturated at higher concentrations of HSA. Comparisons of the uptake clearances in the presence and absence of albumin were performed with Student's $t$ test. $* P<0.05$ (significantly different from control); ${ }^{* *} P<0.01$ (significantly different from control).
Weisiger et al. (1981), who were the first to propose the albumin receptor theory.

The unbound concentrations were determined using the equilibrium dialysis method. The $P S_{\text {inf }}$ value of ANS decreased markedly as the concentration of BSA increased, in association with the decrease in the unbound fraction. In contrast, the $P S_{\mathrm{u} \text {,inf }}$ value of ANS was enhanced markedly as the concentration of BSA increased, although the unbound concentration was kept at approximately $2.4 \mu \mathrm{M}$ (Fig. 5B). In other words, the $P S_{\mathrm{u}, \mathrm{inf}}$ value in the presence of albumin was much higher than could be accounted for by the unbound concentration. The extent of the enhancement in the $P S_{\mathrm{u} \text {,inf }}$ values was saturated at the highest concentration of BSA. These kinetic features are distinctive of the albumin-mediated hepatic transport phenomenon (Weisiger et al., 1981; Forker and Luxon, 1983).

Alternatively, ANS uptake by primary cultured hepatocytes was determined in the presence of varying BSA concentrations, and the ANS concentration was kept constant at $20 \mu \mathrm{M}$ under linear kinetic conditions. As shown in Fig. 6, the $P S_{\text {inf }}$ values decreased as BSA concentration increased, whereas the $P S_{\mathrm{u}}$,inf values increased consistently with those in the uptake experiments under conditions in which the unbound concentration of ANS was kept constant. We determined the albumin-mediated hepatic uptake under two experimental conditions: 1) ANS and BSA concentrations were varied as unbound ANS concentration was kept constant, or 2) the ANS concentration was kept at $20 \mu \mathrm{M}$, although the BSA concentrations were increased. Both experimental conditions demonstrated similar relationships between the albumin concentration and $P S_{\mathrm{u} \text {,inf }}$ enhancement, as shown below (Fig. 8A).

Initial Uptake Rate of PTV by Isolated Human Hepatocytes in the Absence and Presence of HSA. The rate of uptake of PTV by isolated human hepatocytes was measured in the absence and presence of HSA, and the results are summarized in Fig. 7 and Table 2. The PTV uptake increased linearly over 1.5 minutes, and the initial uptake rate was determined from the uptake over $0.25-1.25$ minutes. Likewise to ANS uptake by primary cultured rat hepatocytes, the $P S_{\text {inf }}$ value was estimated by dividing the uptake rate by the initial concentration. The $P S_{\text {inf }}$ value of PTV decreased substantially as the concentration of HSA increased. In contrast, the $P S_{\mathrm{u} \text {,inf }}$ value clearly increased as the HSA concentration increased, and the $P S_{\mathrm{u} \text {,inf }}$ values were saturated at $1 \%$ (w/v) HSA. Therefore, the albumin-mediated hepatic transport phenomenon was also observed in the uptake of PTV by human hepatocytes, known to be mediated by OATP1B1 (Watanabe et al., 2010).

Analysis by the Previous Facilitated-Dissociation Model of the Albumin Dependency of the Ratio of the Unbound Uptake Clearance in the Presence of Albumin to That in Its Absence (R). The facilitated-dissociation model is well known to describe adequately the hepatic uptake rate of a highly bound ligand in the presence of albumin (Forker and Luxon, 1981, 1983; Tsao et al., 1988a). We therefore performed curve fitting of the albumin concentrationdependency of the $R$ values (Fig. 8), on the basis of eq. 12. The facilitated-dissociation model provided adequately fitting curves for the albumin dependency (Fig. 8). The relative capacity for the interaction of albumin with the surface of hepatocytes $\left(r \cdot B_{\max }\right)$ and the dissociation constant of ANS from the bound albumin at the surface $\left(K_{\mathrm{d}, \mathrm{m}}\right)$ were estimated to be $3.51 \pm 1.07$ and $24.4 \pm 17.5 \mu \mathrm{M}$ ( \pm calculated S.D.), respectively (Table 3 ). The values of $r \cdot B_{\max }$ and $K_{\mathrm{d}, \mathrm{m}}$ for PTV were estimated to be $48.8 \pm 11.6$ and $199 \pm 61 \mu \mathrm{M}$ ( \pm calculated S.D.), respectively (Table 3 ). As an alternative, we analyzed the albumin concentration-dependency of the $R$ values using the model developed by Forker and Luxon (1981, 1983). As shown in Supplemental Fig. 1, this model also provided adequately fitting curves for the albumin dependency. The values of $B_{\max }$ and $K_{\mathrm{d}, \mathrm{m}}$ for ANS were estimated to be $3.71 \pm 1.22$ and $31.8 \pm 22.0 \mu \mathrm{M}$ ( \pm calculated S.D.), respectively. The values of $B_{\max }$ and $K_{\mathrm{d}, \mathrm{m}}$ values for PTV were estimated to be $58.7 \pm 21.9$ and $275 \pm 131 \mu \mathrm{M}$ ( \pm calculated S.D.), respectively (Supplemental Table I).

Extrapolation of the In Vivo Hepatic Uptake Clearance from the In Vitro Hepatic Uptake Clearance Obtained Using Isolated Hepatocytes. The in vivo hepatic uptake clearances of unbound ligand for ANS and PTV ( $P S_{\mathrm{u} \text { inf }}^{\mathrm{vitr}}$ ) were extrapolated from the in vitro hepatic uptake clearance obtained using isolated hepatocytes, and the results are summarized in Table 3. The values of $P S_{\mathrm{u} \text {,inf }}^{\text {vito }}$ for ANS and PTV were estimated to be $263 \pm 22$ and $83.0 \pm 20.3 \mathrm{ml} / \mathrm{min}$ per kilogram, respectively. The overall in vivo hepatic intrinsic clearances $\left(C L_{\mathrm{h}, \mathrm{u}, \text { int,all }}^{\text {vivo }}\right)$ for the unbound ligands were also determined from eqs. 14-17 to be

TABLE 2

Relationship between albumin concentration (unbound fraction) and $\mathrm{PS}_{\mathrm{u}, \text { inf }}$ of PTV

Data shown in Fig. 7. PTV uptakes were determined with varying HSA concentrations and PTV concentration held constant at $5 \mu \mathrm{M}$

\begin{tabular}{lccccc}
\hline \multicolumn{1}{c}{ HAS $(\%)$} & 0 & 0.125 & 0.25 & 0.50 & 1.0 \\
\hline Unbound fraction & 1 & 0.237 & 0.0929 & 0.0413 & 0.0182 \\
$P S_{\mathrm{u}, \text { inf }}\left(\mu \mathrm{l} / \mathrm{min}\right.$ per $10^{6}$ cells $)$ & $26.9 \pm 6.6$ & $52.6 \pm 13.3$ & $85.8 \pm 20.4$ & $109 \pm 41$ & $170 \pm 60$ \\
\hline
\end{tabular}


TABLE 3

Extrapolation of the in vivo hepatic uptake clearance from the uptake clearance obtained using isolated hepatocytes and its comparison with the in vivo hepatic overall intrinsic clearance

$P S_{\mathrm{u}, \text { inf }}$, the hepatic uptake clearance in the absence of the albumin by isolated hepatocytes; $P S_{\mathrm{u} \text { inf }}^{\text {vitro }}$, the in vivo hepatic uptake clearance extrapolated from the in vitro hepatic uptake by isolated hepatocytes; $\mathrm{f}_{\mathrm{B}}$, unbound fraction in the blood; $C L_{\mathrm{h}, \mathrm{B}}$, the hepatic clearance for the blood; $C L_{\mathrm{h}, \mathrm{u} \text {,int,all }}^{\text {vivo }}$, the in vivo hepatic overall intrinsic clearance; $R_{\mathrm{vivo} / \text { vitro }}$, the ratio of $C L_{\mathrm{h}, \mathrm{u} \text {,int,all }}^{\text {vivo }} P S_{\mathrm{u}, \text { inf }}^{\text {vitro }}$.

\begin{tabular}{|c|c|c|c|c|c|c|c|c|c|}
\hline \multirow{2}{*}{ Ligands } & \multirow{2}{*}{$P S_{\mathrm{u}, \mathrm{inf}}{ }^{a}$} & \multirow{2}{*}{$P S_{\mathrm{u}, \text { inf }}^{\mathrm{vitro} b}$} & \multirow{2}{*}{$f_{\mathrm{B}}^{c}$} & \multicolumn{3}{|c|}{ Estimated Parameters by the Facilitated-Dissociation Model $^{d}$} & \multirow{2}{*}{$C L_{\mathrm{h}, \mathrm{B}}^{e}$} & \multirow{2}{*}{$C L_{\mathrm{h}, \mathrm{u}, \text { int,all }}^{\mathrm{vivo}}$} & \multirow{2}{*}{$R_{\text {vivo/vitro }}$} \\
\hline & & & & $r \cdot B_{\max }$ & $K_{\mathrm{d}, \mathrm{m}}$ & $\boldsymbol{R}_{\mathbf{5} \%}$ & & & \\
\hline & $\begin{array}{c}\mu \mathrm{l} / \mathrm{min} \\
\text { per } 10^{6} \mathrm{cells}\end{array}$ & $\begin{array}{c}\mathrm{ml} / \mathrm{min} \\
\text { per } \text { kilogram }\end{array}$ & & & & & $\begin{array}{c}\mathrm{ml} / \mathrm{min} \\
\text { per kilogram }\end{array}$ & $\begin{array}{c}\mathrm{ml} / \mathrm{min} \\
\text { per kilogram }\end{array}$ & \\
\hline ANS & $54.8 \pm 4.5^{g}$ & $263 \pm 22$ & 0.00796 & $3.51 \pm 1.07^{h}$ & $24.4 \pm 17.5^{h}$ & 2.70 & 6.43 & 867 & 3.30 \\
\hline PTV & $26.9 \pm 6.6$ & $83.0 \pm 20.3$ & 0.00933 & $48.8 \pm 11.6^{h}$ & $199 \pm 61^{h}$ & 10.6 & 9.64 & 1520 & 18.3 \\
\hline
\end{tabular}

${ }^{a}$ Data shown in Figs 5-7.

${ }^{b}$ Calculated using the following physiologic scaling factors: $1.2 \times 10^{8}$ cells/g of liver, $40 \mathrm{~g}$ liver $/ \mathrm{kg}$ body weight for rat, $25.7 \mathrm{~g}$ liver/kg body weight for human (Davies and Morris, 1993 ).

${ }^{c}$ Calculated by dividing the unbound fraction in the plasma by the blood-to-plasma concentration ratio (Chung et al., 1990a; Watanabe et al., 2010).

${ }^{d}$ The parameters estimated by the fitting curves in Fig. $8 ; r \cdot B_{\max }$, the relative capacity for the interaction of albumin with the surface of hepatocytes; $K_{\mathrm{d}, \mathrm{m}}$, the dissociation constant of the bound albumin from the surface; $R_{5 \%}, R$ value in the presence of $5 \%$ albumin.

${ }^{e}$ Values from the literature (Chung et al., 1990a; Watanabe et al., 2010).

${ }^{f}$ Calculated using a dispersion model as described previously (Roberts and Rowland, 1986; Iwatsubo et al., 1996).

${ }^{g}$ Converted the unit of $P S_{\mathrm{u}, \text { inf }}$ to microliters per minute per $10^{6}$ cells using a scaling factor of $1.1 \times 10^{6}$ cells $/ \mathrm{mg}$ protein

${ }^{h} \pm$ calculated S.D.

867 and $1520 \mathrm{ml} / \mathrm{min}$ per kilogram, respectively. The ratios of $C L_{\mathrm{h}, \mathrm{u}, \text { int,all }}^{\text {vivo }}$ to $P S_{\mathrm{u} \text {,inf }}^{\text {vito }}$ for ANS and PTV were estimated to be 3.30 and 18.3, respectively, indicating a poor predictive accuracy by IVIVE. The $R$ values in the range of physiologic concentrations of albumin were 2.70 and 10.6, respectively, which are close to the ratios of $C L_{\mathrm{h}, \mathrm{u}, \text { int,all }}^{\mathrm{viv}}$ to $P S_{\mathrm{u}, \text { inf }}^{\text {vitro }}$.

\section{Discussion}

In this study, we used primary cultured rat hepatocytes and isolated human hepatocytes to determine whether BSA and HSA enhanced the $P S_{\mathrm{u} \text {,inf }}$ of ANS and PTV, respectively. These organic anions have been shown to be taken up by the liver via a transporter-mediated process (Sugiyama et al., 1983; Chung et al., 1990a,b; Watanabe et al., 2010, 2011; Kimoto et al., 2012). As shown in Figs. 5 and 6, the presence of albumin prominently enhanced $P S_{\mathrm{u} \text {,inf }}$ values of hepatocytes. This enhancement of the $P S_{\mathrm{u} \text {,inf }}$ values was saturated at high concentrations of albumins, indicating that it was the albumin-mediated hepatic uptake phenomenon being observed in rat and human hepatocytes (Figs. 5B and 6B).

As discussed earlier, several mechanisms have been proposed to account for the enhancement of the hepatic uptake clearance of unbound ligand. In the present study, the albumin-mediated hepatic uptake transport of ANS and PTV was analyzed using a facilitated-dissociation model in which contact of the ligand-albumin complex with the surface of hepatocytes enhanced the dissociation of the ligand from the complex and provided the hepatocytes with more unbound ligand for uptake (Fig. 2). We therefore performed curve fitting using the governing equation, eq. 12. The calculated line fit well with the data for the enhancement ratios of both ANS and PTV uptake (Fig. 8). The values of $r \cdot B_{\max }$ and $K_{\mathrm{d}, \mathrm{m}}$ for ANS were estimated to be 4 and $20 \mu \mathrm{M}$, respectively. The values of $r \cdot B_{\max }$ and $K_{\mathrm{d}, \mathrm{m}}$ for PTV were estimated to be 50 and $200 \mu \mathrm{M}$, respectively. These values of $K_{\mathrm{d}, \mathrm{m}}$ were compatible with those reported in other studies (Weisiger et al., 1981; Forker and Luxon, 1983; Elmadhoun et al., 2001). Various models previously proposed can explain the kinetic features; the albumin-dependent enhancement of $P S_{\mathrm{u}, \text { inf }}$ and its saturation in the presence of higher concentrations of albumin and provide the enhancement ratio of $P S_{\mathrm{u} \text {,inf }}$ in the presence of physiologic concentrations of albumin, although it is difficult to distinguish the most adequate of the various models. Among these models, the facilitated-dissociation model developed by Forker and Luxon (1983) was most similar to a model whose basis is the albumin receptor theory. The model also provided a good prediction of the relationship between the enhancement of $P S_{\mathrm{u}, \text { inf }}$ and the albumin concentrations (Supplemental Fig. 1), with $K_{\mathrm{d}, \mathrm{m}}$ values of 30 and $300 \mu \mathrm{M}$, respectively (Supplemental Table I), compatible with our

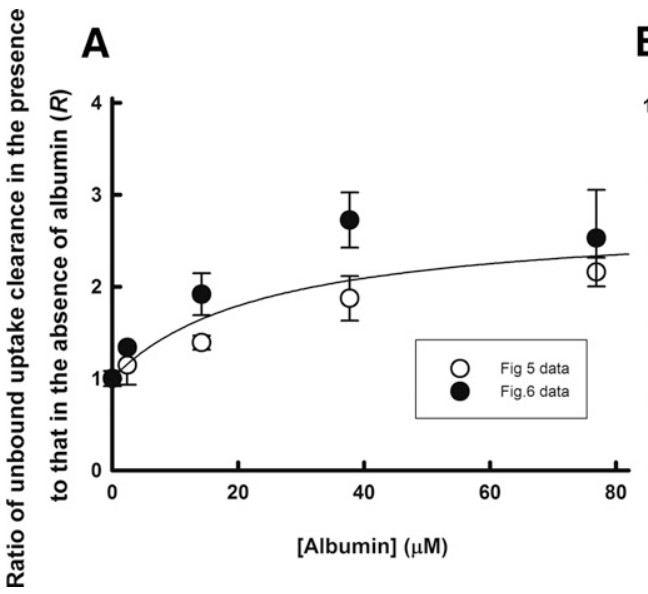

B

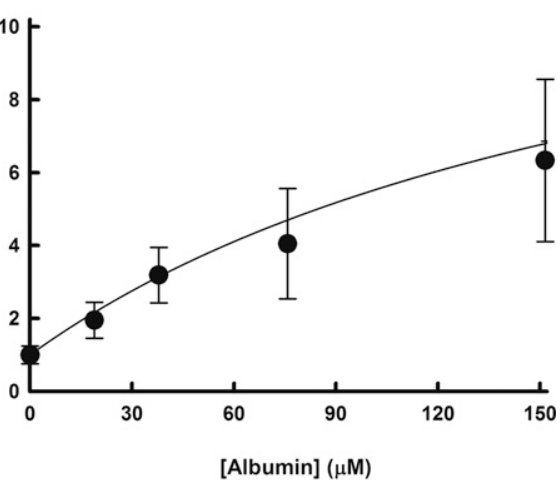

Fig. 8. Curve fitting of the relationship between the albumin concentration and the $R$ values for ANS uptake (A) and PTV uptake (B) analyzed by the facilitated-dissociation model. The $R$ values for ANS and PTV were estimated from the data shown in Figs. 5 and 6 and Fig.7, respectively. The values of $\frac{n}{K_{\mathrm{d}}}$ for ANS and PTV were estimated by the equilibrium dialysis method to be 0.501 and $0.248 \mu \mathrm{M}^{-1}$, respectively. The relationships were fitted to eq. 12 using a nonlinear least-squares method. The fitting curve for ANS and PTV adequately predicted the observed data. The lines in the figures show the fitted lines determined by analysis using the facilitateddissociation model. 
model. Either mechanism can explain the kinetic features of the albumin-mediated hepatic uptake and the enhancement ratio of $P S_{\mathrm{u}, \text { inf }}$ value under physiologic levels of albumin.

Blitzer and Lyons (1985) directly demonstrated, using rat basolateral liver plasma membrane vesicles, that albumin enhanced $\mathrm{Na}^{+}$-dependent bile acid uptake. They proposed as a possible mechanism that the binding of albumin-bile acid complex to the liver plasma membrane might facilitate the interaction of bile acid with its transporter and enhance the bile acid available for uptake, implying that the bile-acid transporter might function as the albumin receptor and facilitate the dissociation of the albumin-bile acid complex. If the transporter were involved in the interaction of albumin with the surface of hepatocytes, this could explain why the $R$ values differed so markedly between ANS and PTV, although their unbound fractions in the blood were similar. According to a kinetic study by Blitzer and Lyons (1985), albumin reduced the bile-acid transporter $K_{\mathrm{m}}$ value; in other words, the binding of albumin to the liver plasma membrane enhanced the affinity of the bile acid for its transporter. PTV shows a relatively high affinity for OATP (Kimoto et al., 2012), whereas ANS shows a relatively low affinity for Oatp (Chung et al., 1990b), leading us to hypothesize that the higher the affinity for the transporter, the more effective is the albumin-mediated enhancement. Further development of another model, including the interaction between the albumin-organic anion complex and the transporter is required to demonstrate its mechanism.

There are several published reports concerning the interaction of albumin with the surface of hepatocytes. Weisiger et al. (1981) demonstrated by using isolated rat hepatocytes directly that ${ }^{125} \mathrm{I}$ labeled albumin binds to the surface of hepatocytes with a dissociation constant of $25 \mu \mathrm{M}$. Horie et al. (1988) demonstrated using spectrophotometric methods (electron spin resonance and absorption spectrum) that the interaction of albumin and isolated rat hepatocytes induces a conformational change in albumin. From another perspective, Burczynski and others, using several proteins including modified albumins, demonstrated that an ionic interaction between extracellular protein and hepatocytes occurs at the cell surface, which enhances palmitate uptake by hepatocytes (Burczynski et al., 2001; Elmadhoun et al., 2001). However, the physiologic and biochemical relevance of this interaction remains unknown, and its mechanism requires further investigation.

Recently, on the basis of the free-drug hypothesis, quantitative IVIVE of hepatic uptake clearance using cryopreserved human hepatocytes resulted in good predictions of the uptake of some organic anions (Watanabe et al., 2010). However, the uptake of other organic anions that bind avidly to serum albumin was poorly predicted (Watanabe et al., 2010, 2011). Hypothesizing that the albumin-mediated hepatic uptake mechanism might be a factor in these poor predictions, we estimated the $R$ values in physiologic albumin concentrations (5\% albumin) on the basis of the facilitated-dissociation model. The $R$ values for ANS and PTV were estimated to be 2.7 and 11 , respectively, implying that the albumin-mediated hepatic uptake mechanism should be considered in vivo. Furthermore, the unbound hepatic uptake clearances $\left(P S_{\mathrm{u}, \text { inf }}^{\text {vitr }}\right)$ for ANS and PTV in vivo (per body weight) were extrapolated from the in vitro unbound hepatic uptake clearances (Davies and Morris, 1993) to be 260 and $83 \mathrm{ml} / \mathrm{min}$ per kilogram, respectively (Table 3 ). The hepatic uptake clearance $\left(C L_{\mathrm{h}, \mathrm{u}, \text { int,all }}^{\text {vivo }}\right)$ was estimated from in vivo kinetic parameters using a dispersion model, as described previously (Roberts and Rowland, 1986; Iwatsubo et al., 1996). The $C L_{\mathrm{h}, \mathrm{u}, \text { int,all }}^{\text {vivo }}$ values were determined to be 870 and $1500 \mathrm{ml} / \mathrm{min}$ per kilogram, respectively. The ratios $\left(R_{\mathrm{vivo} / \text { vitro }}\right)$ of $C L_{\mathrm{h}, \mathrm{u} \text {,int,all }}^{\text {vio }} P S_{\mathrm{u} \text {,inf }}^{\text {vitro }}$ for $\mathrm{ANS}$ and PTV were estimated to be 3 and 18 , respectively, indicating that the huge discrepancy seen in IVIVE could be attributed mainly to albuminmediated hepatic uptake and could be largely corrected by using the $R$ value estimated with the facilitated-dissociation model. In the present study, however, IVIVE taking into account the albumin-mediated hepatic uptake provided good predictions for only the two organic anions ANS and PTV; therefore, further investigations of other organic anions that are highly bound to albumin are required to elucidate how albumin-mediated hepatic uptake might be involved in IVIVE.

Alternative reasons also could be considered to raise poor predictions of IVIVE: 1) $\mathrm{pH}$ difference between intracellular and extracellular spaces (Poulin and Haddad, 2015), 2) the concentration difference in between the plasma and liver (Poulin et al., 2016), 3) the activity heterogeneity of the human cryopreserved hepatocytes (Badolo et al., 2011), and 4) the kinetic model describing the behavior of anion in the liver (Iwatsubo et al., 1996). Poulin et al. (2016) developed a useful procedure for quantifying the unbound ligand concentration under in vivo conditions by taking into account $\mathrm{pH}$ difference between intracellular and extracellular spaces and the concentration difference in between the plasma and liver. This empirical method provides an improvement of quantitative IVIVE for a range of drugs that bind avidly to albumin (Poulin et al., 2012, 2016; Poulin and Haddad, 2015). Very recently, Bounakta et al. (2018) and Poulin et al. (2017) demonstrated using an isolated perfused rat liver system that the IVIVE method whose basis was the albumin-mediated uptake mechanism provided the most accurate predictions of in vivo hepatic clearance compared with other empirical IVIVE considering $\mathrm{pH}$ and albumin concentration differences between the plasma and liver. Thus, it is important to note that a kinetic model for describing the albumin-mediated hepatic uptake phenomenon for a ligand avidly bound to albumin is indispensable for its robust IVIVE.

In conclusion, we have demonstrated that the unbound hepatic uptake clearance of the organic anions ANS and PTV was substantially enhanced by the presence of albumin and that this enhancement was saturated at a high concentration of albumin. Therefore, albuminmediated hepatic transport was demonstrated to play a role in uptake of these anions by rat and human hepatocytes. The relationship between the enhancement of the unbound hepatic clearance and the albumin concentration could be accurately predicted by the facilitateddissociation model constructed previously, in which the interaction of the ligand-albumin complex with the cell surface enhances the dissociation of the complex to make unbound ligand available for uptake to the hepatocytes. By considering this enhancement of hepatic uptake clearance by albumin, we could also predict the uptake clearance in vivo from that observed in isolated hepatocytes. In light of these findings, we propose that the facilitated-dissociation model can be applied to describing the phenomenon of albumin-mediated hepatic uptake via organic anion transporters and to evaluating hepatic uptake clearance in vivo.

\section{Authorship Contributions}

Participated in research design: Miyauchi, Masuda, Kim, Shimono, Sugiyama.

Conducted experiments: Miyauchi, Masuda, Kim, Yuudai Tanaka, Iwakado, Nemoto, Sasaki, Lee.

Performed data analysis: Miyauchi, Masuda, Kim, Lee, Shimono, Sasaki, Sugiyama.

Wrote or contributed to the writing of the manuscript: Miyauchi, Kim, Yoshio Tanaka, Sugiyama.

\section{References}

Badolo L, Trancart MM, Gustavsson L, and Chesné C (2011) Effect of cryopreservation on the activity of OATP1B $1 / 3$ and OCT1 in isolated human hepatocytes. Chem Biol Interact 190: $165-170$.

Bass L and Pond SM (1988) The puzzle of rates of cellular uptake of protein-bound ligands, in Pharmacokinetics: Mathematical and Statistical Approaches to Matabolism and Distribution of Chemicals and Drugs (Pecile A and Rescigno A eds) pp 245-269, Plenum, London. 
Blitzer BL and Lyons L (1985) Enhancement of Na+-dependent bile acid uptake by albumin: direct demonstration in rat basolateral liver plasma membrane vesicles. Am J Physiol 249: G34-G38.

Bounakta S, Bteich M, Mantha M, Poulin P, and Haddad S (2018) Predictions of bisphenol A hepatic clearance in the isolated perfused rat liver (IPRL): impact of albumin binding and of co-administration with naproxen. Xenobiotica 48:135-147.

Burczynski FJ, Cai ZS, Moran JB, and Forker EL (1989) Palmitate uptake by cultured hepatocytes: albumin binding and stagnant layer phenomena. Am J Physiol 257:G584-G593.

Burczynski FJ, Wang GQ, Elmadhoun B, She YM, Roberts MS, and Standing KG (2001) Hepatocyte $[3 \mathrm{H}]$-palmitate uptake: effect of albumin surface charge modification. Can J Physio Pharmacol 79:868-875.

Chung YB, Miyauchi S, Sugiyama Y, Harashima H, Iga T, and Hanano M (1990a) Effect of various organic anions on the plasma disappearance of 1-anilino-8-naphthalene sulfonate. $J$ Hepatol 11:240-251.

Chung YB, Miyauchi S, Sugiyama Y, Harashima H, Iga T, and Hanano M (1990b) Kinetic analysis of the dose-dependent hepatic handling of 1-anilino-8-naphthalene sulfonate in rats. J Pharmacokinet Biopharm 18:313-333.

Davies B and Morris T (1993) Physiological parameters in laboratory animals and humans. Pharm Res 10:1093-1095.

Elmadhoun BM, Wang GQ, Kirshenbaum LA, and Burczynski FJ (2001) Palmitate uptake by neonatal rat myocytes and hepatocytes. Role of extracellular protein. Eur J Biochem 268: $3145-3153$.

Forker EL and Luxon BA (1981) Albumin helps mediate removal of taurocholate by rat liver. $J$ Clin Invest 67:1517-1522.

Forker EL and Luxon BA (1983) Albumin-mediated transport of rose bengal by perfused rat liver. Kinetics of the reaction at the cell surface. J Clin Invest 72:1764-1771.

Forker EL, Luxon BA, Snell M, and Shurmantine WO (1982) Effect of albumin binding on the hepatic transport of rose bengal: surface-mediated dissociation of limited capacity. J Pharmacol Exp Ther 223:342-347.

Horie T, Mizuma T, Kasai S, and Awazu S (1988) Conformational change in plasma albumin due to interaction with isolated rat hepatocyte. Am J Physiol 254:G465-G470.

Ichikawa M, Tsao SC, Lin TH, Miyauchi S, Sawada Y, Iga T, Hanano M, and Sugiyama Y (1992) 'Albumin-mediated transport phenomenon' observed for ligands with high membrane permeability. Effect of the unstirred water layer in the Disse's space of rat liver. J Hepatol 16:38-49.

Iwatsubo T, Hirota N, Ooie T, Suzuki H, and Sugiyama Y (1996) Prediction of in vivo drug disposition from in vitro data based on physiological pharmacokinetics. Biopharm Drug Dispos 17:273-310.

Kimoto E, Yoshida K, Balogh LM, Bi YA, Maeda K, El-Kattan A, Sugiyama Y, and Lai Y (2012) Characterization of organic anion transporting polypeptide (OATP) expression and its functional contribution to the uptake of substrates in human hepatocytes. Mol Pharm 9:3535-3542.

Masuda M, Ichikawa Y, Shimono K, Shimizu M, Tanaka Y, Nara T, and Miyauchi S (2014) Electrophysiological characterization of human $\mathrm{Na}^{+} /$taurocholate cotransporting polypeptide (hNTCP) heterologously expressed in Xenopus laevis oocytes. Arch Biochem Biophys 562. $115-121$.

Meijer DK and van der Sluijs P (1989) Covalent and noncovalent protein binding of drugs: implications for hepatic clearance, storage, and cell-specific drug delivery. Pharm Res 6 $105-118$

Miyauchi S, Sawada Y, Iga T, Hanano M, and Sugiyama Y (1993) Comparison of the hepatic uptake clearances of fifteen drugs with a wide range of membrane permeabilities in isolated rat hepatocytes and perfused rat livers. Pharm Res 10:434-440.

Pond SM, Davis CKC, Bogoyevitch MA, Gordon RA, Weisiger RA, and Bass L (1992) Uptake of palmitate by hepatocyte suspensions: facilitation by albumin? Am J Physiol 262:G883-G894.

Poulin P, Bteich M, and Haddad S (2017) Supplemental analysis of the prediction of hepatic clearance of binary mixtures of bisphenol A and naproxen determined in an isolated perfused rat liver model to promote the understanding of potential albumin-facilitated hepatic uptake mechanism. J Pharm Sci 106:3207-3214.

Poulin P, Burczynski FJ, and Haddad S (2016) The role of extracellular binding proteins in the cellular uptake of drugs: impact on quantitative in vitro-to-in vivo extrapolations of toxicity and efficacy in physiologically based pharmacokinetic-pharmacodynamic research. J Pharm Sci 105 : 497-508.

Poulin P and Haddad S (2015) Albumin and uptake of drugs in cells: additional validation exercises of a recently published equation that quantifies the albumin-facilitated uptake mechanism(s) in physiologically based pharmacokinetic and pharmacodynamic modeling research. J Pharm Sci 104:4448-4458.

Poulin P, Hop CE, Ho Q, Halladay JS, Haddad S, and Kenny JR (2012) Comparative assessment of In Vitro-In Vivo extrapolation methods used for predicting hepatic metabolic clearance of drugs. J Pharm Sci 101:4308-4326.

Roberts MS and Rowland M (1986) A dispersion model of hepatic elimination: 1. Formulation of the model and bolus considerations. J Pharmacokinet Biopharm 14:227-260.

Shand DG, Cotham RH, and Wilkinson GR (1976) Perfusion-limited of plasma drug binding on hepatic drug extraction. Life Sci 19:125-130.

Sugiyama Y, Kimura S, Lin JH, Izukura M, Awazu S, and Hanano M (1983) Effects of organic anions on the uptake of 1-anilino-8-naphthalenesulfonate by isolated liver cells. J Pharm Sci 72: 871-876.

Tsao SC, Sugiyama Y, Sawada Y, Iga T, and Hanano M (1988a) Kinetic analysis of albuminmediated uptake of warfarin by perfused rat liver. J Pharmacokinet Biopharm 16:165-181.

Tsao SC, Sugiyama Y, Shinmura K, Sawada Y, Nagase S, Iga T, and Hanano M (1988b) Protein mediated hepatic uptake of rose bengal in analbuminemic mutant rats (NAR). Albumin is no indispensable to the protein-mediated transport of rose bengal. Drug Metab Dispos 16:482-489.

van der Sluijs P, Postema B, and Meijer DK (1987) Lactosylation of albumin reduces uptake rate of dibromosulfophthalein in perfused rat liver and dissociation rate from albumin in vitro. Hepatology 7:688-695.

Watanabe T, Kusuhara H, Maeda K, Kanamaru H, Saito Y, Hu Z, and Sugiyama Y (2010) Investigation of the rate-determining process in the hepatic elimination of HMG-CoA reductase inhibitors in rats and humans. Drug Metab Dispos 38:215-222.

Watanabe T, Kusuhara H, Watanabe T, Debori Y, Maeda K, Kondo T, Nakayama H, Horita S Ogilvie BW, Parkinson A, et al. (2011) Prediction of the overall renal tubular secretion and hepatic clearance of anionic drugs and a renal drug-drug interaction involving organic anion transporter 3 in humans by in vitro uptake experiments. Drug Metab Dispos 39:1031-1038.

Weisiger R, Gollan J, and Ockner R (1981) Receptor for albumin on the liver cell surface may mediate uptake of fatty acids and other albumin-bound substances. Science 211:1048-1051.

Weisiger RA (1985) Dissociation from albumin: a potentially rate-limiting step in the clearance of substances by the liver. Proc Natl Acad Sci USA 82:1563-1567.

Weisiger RA, Pond S, and Bass L (1991) Hepatic uptake of protein-bound ligands: extended sinusoidal perfusion model. Am J Physiol 261:G872-G884.

Weisiger RA, Zacks CM, Smith ND, and Boyer JL (1984) Effect of albumin binding on extraction of sulfobromophthalein by perfused elasmobranch liver: evidence for dissociation-limited uptake. Hepatology 4:492-501

Wilkinson GR and Shand DG (1975) Commentary: a physiological approach to hepatic drug clearance. Clin Pharmacol Ther 18:377-390.

Address correspondence to: Dr. Yuichi Sugiyama, Sugiyama Laboratory, RIKEN Innovation Center, RIKEN Cluster for Industry Partnerships, RIKEN, 1-7-22 Suehiro-cho, Tsurumi-ku, Yokohama 230-0045, Japan. E-mail: ychi.sugiyama@ riken.jp 